\title{
Naturalistic artistic decision-making and metacognition in the music studio
}

\author{
M. Nyssim Lefford ${ }^{1}$ (1) - Paul Thompson ${ }^{2}$
}

Received: 25 December 2017 / Accepted: 7 June 2018 / Published online: 15 June 2018

(c) The Author(s) 2018

\begin{abstract}
Professional artistic contexts, such as studio-based music production, are rarely investigated in naturalistic decision-making (NDM) research, though creative work is characterised by uncertainty, risk, a lack of clearly definable goals, and in the case of music production, a complex socio-technical working environment that brings together a diverse group of specialized collaborators. This study investigates NDM in the music production studio. In music production, there is a professional role explicitly tasked with taking decisions - the (record) producer. The producer, as a creative collaborator, is differentiated as a problem-solver, solution creator and goal setter. This investigation looks at the producer's metacognitive abilities for reflecting on the nature of problems and decisions. An important challenge for this study is to develop methods for observing decision-making without unrealistically reducing the amount of uncertainty around outcomes or creative intention within a studio production. In the face of that, a method is proposed that combines socio-cultural musicology and cognitive approaches and uses ethnographic data. Preliminary findings shed light on how the producer in this study self-manages his decisions and his interactions with, and in response to, the production environment; how decisions and actions sustain collaboration; how experience is utilized to identify scenarios and choose actions; and the kinds of strategies employed and their expected outcomes. Findings provide evidence that exercising producing skills and performing production tasks involve metacognitive reflection.
\end{abstract}

Keywords Naturalistic decision-making $\cdot$ Artistic decision-making $\cdot$ Metacognition $\cdot$ Sociomusicology $\cdot$ Music production

\section{Introduction}

Artistic working environments are underrepresented in the naturalistic decision-making (NDM) literature, and although they are methodologically challenging to investigate, artistic domains provide interesting scenarios for the study of NDM. Professional artistic contexts, such as studio-based music production, are particularly interesting because of the constraints professional work demands. In some regard, the recording studio is similar to a professional design studio where, "[c]ollaboration between people, especially in teams,

M. Nyssim Lefford

nyssim.lefford@1tu.se

Paul Thompson

P.A.Thompson@leedsbeckett.ac.uk

1 Audio Technology Program, Luleå University of Technology, Piteå, Sweden

2 Music, Sound and Performance, Leeds Beckett University, Leeds, UK requires communication, about intentions, ideas, visions and knowledge." (Vyas et al. 2013, p. 415). A central difference, however, is the context in which a popular music recording is created, as decisions are often taken within poorly defined parameters from the outset. The popular music recording emerges from the complex and direct interaction between the recording's contributors. The following study investigates NDM in the music production studio to increase understanding of how this complex work proceeds.

When viewed through the filters of NDM, music production inside the recording studio bears many similarities with more widely studied, less creative, areas such as emergency medical services, fire-fighting and aviation. In a studio production, the work is often performed under difficult conditions. There is an ever-present pressure to perform exceptionally well. The stress of the working context connects this research to many other macrocognition studies, but may also distinguish music production somewhat from design, which usually does not deal with the same time constraints. While (hopefully) neither lives 
nor property are at stake, working professionally in the context of a recording studio involves significant personal risk. Professional reputations are particularly vulnerable as stakeholders bring competing interests. Those who finance recording projects, such as record companies, typically impose stringent time and resource constraints on studio productions and employ representatives to verify that the musical product meets their market-based needs. Artistic collaborators are similarly protective of their personal economic interests, but their interests are tied to artistic integrity. In this situation, artistic collaborators and financial stakeholders can, at times, find themselves at odds with each other. Music productions involve: "negotiating the complex and sometimes contradictory demands of creativity, commerce, and culture." (Neuenfeldt in Green and Porcello 2005, p. 89) and demand personal investment of time, resources, creativity and originality. As a result, participants can often feel that their unique talents and thus reputations (and thus careers) are determined by the amount of agency they have within the process and by the decisions that are taken during a production. All of these factors contribute to the intense working environment of the recording studio. Emotional stress and anxiety can run high, and this inevitably impacts how production decisions are taken. Because the commercial recording studio is a professional environment, in practice, those factors are naturalistically balanced with more pragmatic, qualifiable and quantifiable concerns.

Uncertainty adds to the inherent stresses and risks of artistic creation. Although the basic format of the work product may be known in the earliest stages, the production of a sound recording involves emergent and contingent properties (as in Simon 1973 and comparable to Klein et al. 2003). In other words, music production proceeds towards the creation of a recorded artefact with scant information about the features that will characterize the final product. The Beatles did not enter the studio to record Sgt. Pepper's Lonely Hearts Club Band (The Beatles 1967) as we know it today. They entered the studio to record a collection of half-finished songs, and at junctures in the creative process, they took decisions about future actions. Production goals, the Beatles' goals, any creators' goals are the least defined and most vague at the start of the production process but, over time, actions and decisions accumulate and impart attributes and defining characteristics upon the emergent artefact. Though there are standard practices and common actions that are taken in music production, every production and every recorded artefact is unique. This is because actions are taken and decisions are made at different times, in different orders and combinations, to yield different, distinctive results and characteristics, and there are no fixed tolerances for variation.

\section{Macrocognitive work and metacognition}

Macrocognition is a term used to describe how cognition emerges in non-laboratory situations and more real-world and natural environments (Cacciabue and Hollnagel 1995). In the natural environment of a commercial recording studio, macrocognitive processes emerge from the interactions between the contributors (such as musicians, engineers and record producers), and the recording and music technologies that are used in creating the musical product. Such arrangements are referred to as macrocognitive work systems (MWSs). Patterson and Hoffman define MWSs as "systems in which people use advanced technology to collaborate for the purpose of conducting work" (Patterson and Hoffman 2012, p. 221). In the studio, technology enables various participants to contribute artistic work. The sharing of artistic work is integral to studio collaboration. Historically, advanced technologies were not required to support collaboration. Collaborators worked together face-to-face in real time. Contemporary productions are increasingly sustained across locations using the Internet and other networking technologies. And, as in any other MWS, "macrocognitive work is carried out under constraints of organizational resources, requirements, and culture" (Patterson and Hoffman 2012, p. 221). The work performed in the recording studio is inseparable from the cultural context established there.

The numerous and varying demands of this macrocognitive setting require contributors inside the recording studio to regulate their thinking using metacognitive skills. Metacognition involves evaluating one's own cognition (Flavell 1987) or thinking processes. During a studio production, individuals can use metacognition as a way of managing the multiple cognitive demands placed upon them during collaboration and the multiple forms of information available for decision-making.

In previous studies on metacognition, Davidson et al. have argued that " $[\mathrm{m}]$ etacognition helps the problem-solver (1) recognize that there is a problem to be solved, (2) figure out what exactly the problem is, and (3) understand how to reach a solution." (Davidson et al. 1994, p. 208). Metacognitive judgments utilize internal resources such as memory, knowledge, prior experience and subjective feelings. Many researchers distinguish between "experience-based and information-based metacognitive judgments" (Koriat and Levy-Sadot 2000). Koriat and Levy-Sadot (2000) further suggest making "a distinction between metacognitive judgments that are based directly on subjective experience (an immediate "gut feeling") and those that are based on the retrieval and weighing of information from memory." (Koriat and Levy-Sadot 2000, p. 193).

In creative contexts, Koriat and Levy-Sadot's use of the word "feeling" is especially problematic because it implies 
an emotional response. In studio-based music production, both the creative process and the artefacts produced are charged with emotional significance. At first glance, it may appear that gut feelings rule creative judgements, but much in music production is reasoned. The balancing and weighting of dissimilar, perhaps seemingly unrelated, types of information is an integral characteristic of any music production decision. This study avoids categorical distinctions between experience and information-based decisions, gut feelings, perceived and/or induced emotions, or readings on the meter of a recording device. All, the reasoned and the emotionally, intuitively felt, could plausibly be (potentially equal) contributors to any given production decision. Some of these types of information may be readily interpreted using cognitive or perceptual approaches and some requires specialized knowledge of music and music production. This study, therefore, aims to investigate metacognition within the macrocognitive environment of the recording studio by combining cognitive and socio-cultural musicological approaches.

\section{The record producer}

What is particularly interesting about music production within the context of NDM is that there is a professional role explicitly tasked with taking decisions-the (record) producer. It is the same decision-making role that gives producers a point of significant interest within socio-cultural music studies because they play a distinct role in coordinating musical collaborations. Since, production work requires collaboration amongst a diverse group of experts, some technical and some artistic, it is not surprising that coordinating is a formal responsibility. Record producers have been described as "technological intermediaries" (Feld 1994, p. 282) in their role between people, performers and recording technologies. This interstitial role further distinguishes music production from design. Though a lead designer may coordinate among collaborators using different technologies, the producer, metaphorically and often literally, physically sits between the performers and the technology that captures performers' work. This too makes them an interesting subject for NDM.

The producer is responsible for managing recording sessions, navigating collaborators through the sociotechnical complexities of the environment; coaching the performances of individual contributors; fostering group cohesion, providing aesthetic guidance, feedback and/or leadership; supplying or fostering innovation; and facilitating technical implementation of recording procedures - all on the way to delivering a recorded product. To perform this work, the producer must be a deft problem detector. Klein et al. (2005) describe problem detection as a kind of "sensemaking". With the producer's expertise and experience comes a special sensitivity and "alertness" to "triggers and alarms" and "indicators" (Klein et al. 2005, p. 21). Of all the collaborators in a production, producers (are expected to) have the most developed "perceptual and conceptual ability to notice subtle signs, the ability to use expectancies the sophistication of mental models, and the experience base that provides a sense of typicality." (Klein et al. 2005, p. 20-21). With all that could go wrong, framing the producer's decision-making is the understanding that ultimately the producer is accountable for the completion and quality of a recording, and ensuring its commercial viability. The producer's problem detection is linked to (personal) risk.

Given the producer's professional profile, it would not be surprising to discover that producers, as compared to other collaborators in a music production, have preternatural (metacognitive) abilities for reflecting on the nature of problems and decisions, and for problem registration, encoding and framing (as in Kirsh 2009), which contributes to their expertise as problem-solvers, solution creators and goal setters. The producer, as a collaborator, is further differentiated by these capacities. The producer's decisions fundamentally influence the aesthetic character of the production (Zak 2001) and, although the finished recorded artefact cannot be characterized at the start, the producer says when the work suffices, and verifies and validates it. Insight into the producer's metacognitive reflections provides insight into the producer's decision-making and problem-solving.

\section{The scope of the study}

A main challenge for this study is to develop methods for observing decision-making without unrealistically reducing the amount of uncertainty around outcomes or creative intention within a studio production; methods that are as informative to socio-cultural musicology as to NDM. Is it possible to observe musical creators directing their own decision-making processes at creative junctures? Is there evidence of metacognitive reflection? The answers to these questions are of interest not only to NDM research, but also to socio-cultural musicology, which seeks to understand the function of certain decisions and interactions amongst musical collaborators. This study presents preliminary findings that both fields of research can build upon, with the central exploratory purpose of using a case study to develop an approach that might later be scaled.

\section{Work, goals, information and production decisions}

Like other domains investigated in NDM research, music production is a sociotechnical practice, and in studio collaborations, disparate forms of expertise are brought together. 
Participants play different instruments or perform specialized technical roles, and so, they vary in experience and function and supply different information for decisions. It is not unusual for multiple collaborators to exude strong and diverging influences over goals and outcomes. Compromises are reached to satisfy aesthetic, commercial and social needs. However, in artistic work, individualistic, idiosyncratic preferences, perceptions and biases overtly weigh on decisions and, importantly, they are often what give work products their value.

Relatedly, an essential property of creative environments is that innovative problem-solving and novelty are highly prized. However, at the same time, professionally produced recorded music products are expected to be commercially viable, or at least in some way, they should professionally enable an artist. Commercial viability imposes normalizing constraints on potential outcomes. Too much innovation or novelty can deter audiences, and so novelty and innovation are valuable and necessary but must also fall within limits. Those limits are defined by the expectations of the musical genre, the personal work history of an artist, and other cultural factors. But, these are not fixed boundaries per se and can change over time in relation to individual and popular taste. The vagueness around these limits is a major source of uncertainty in production.

Recording sessions involve a lot of discussion about what is to be performed or done and how. There may be rehearsals or experiments to test a course of action before it is executed for recording. Once musical parts have been recorded, time may be allocated for mixing or processing recorded material. These in-between times can last minutes or days, sometimes weeks. Clearly, these intervals provide the producer with opportunities to analyse materials and decisions, compare outcomes and weigh options, as in classical decision-making models. For example, the producer might decide to dedicate session time to engineering the sound of the snare drum sound. With no other decisions to make in that moment, a producer listens critically and compares what is heard to what is known about typical snare drum sounds in the genre within which (s)he is working. In practice, the decision about the sound is not a simple exercise in comparison. The producer would consider the client/artist's goals, aesthetics and commercial viability as well. Nevertheless, such scenario may plausibly be deconstructed into a set of potential outcomes that are ascribed values.

Some decisions, taken at some times, may intuitively feel and indeed (by some metrics) be less risky than others, depending on constraints, resources, available expertise, expectations for the production and the culture of collaboration. Rogalski (1999), in her studies of decision-makers managing dynamic risk, distinguishes between decisionmaking "stricto sensu" and "lato sensu". Each engages distinct cognitive processes. Stricto sensu decision-making may be defined more classically as decisions that are "the choice between options in the presence of possible events". The decision-maker subjectively estimates the probability of events and weights them according to subjective values. A lato sensu decision "involves situation assessment, and elaboration of possible actions" (Rogalski 1999, p. 248).

Producers do at times decide and react under time-constrained, high-pressure conditions; utilizing experience to identify and categorize situations, as in Klein's Recognition Primed Decision Making (RPD) (Klein 1993). For the same snare drum assessment described above, the producer might instead use a reference recording and try to match through technical means the sound currently being recorded to a template provided by a commercially successful recording. The upshot of this would be to make the sound typical of the genre. At the same juncture, the producer might recognize the similarities between the to-be-recorded sound and the reference, and still without using any sort of classical analysis of outcomes, choose to depart from the template. In such situations, metacognitive reflection is one way to explain this decision. Importantly, in both cases, the choice of template pre-supposes some form of meta-analysis.

Numerous forms of information are available to the producer for these decisions. Much of it is observed directly through the recording technology itself, through meters on recording equipment, by monitoring audio through loudspeakers, and hearing changes to the sound as technologies are utilized and as music is performed. The producers' prior experiences are influences on decisions, including knowledge of recorded music history, specific genres and culture and art generally; awareness of existing social pressures at large, outside the production; and formal training (if any). In this study, we have treated experience as a special kind of (privileged) information, subjectively acquired certainly, but as one type of information factored into decisions that integrate many varied forms of information. However, the nature of experience, how it provides a knowledge base for the producer or the affective influence of that information are all beyond the scope of this investigation. Our study does not attempt to observe the intricate workings of given metacognitive reflections, but rather metacognitive reflection's role in the producer's decisions. The skillset associated with professional producers, in practice, suggests that certain types of decisions are the producer's responsibility. Any metacognitive reflection associated with those decisions would then be part of a production practice. Producers have:

- Specialized perceptual skills to detect (auditory and musical) features in the recording and make fine discriminations among features.

- Analytical skills to deconstruct and parameterize attributes of recordings and musical performances. 
- Abilities to ascribe value to attributes of recordings and musical performances.

- And, therefore, metacognitive skills to choose appropriate ways to determine what is an interesting or valuable perceptual feature within a given context.

- Skills to make comparisons (e.g. how is this product, decision or situation like/dislike similar products, decisions or situations?).

- Metacognitive abilities to recognize influences, biases, effects and contingencies and factor them into or out of decisions.

- Procedural knowledge, what to do technically, musically, managerially, etc.

- Abilities to ascribe value to actions and decisions.

- Metacognitive skills to discern what type of problemsolving and procedural knowledge should be applied and when.

- Cognitive skills to function in a social context, and identify significant happenings.

- Abilities to sustain meaningful communications with others holding differing expertise, and thus able to establish a shared context.

- Abilities to make inferences about the behaviours of others, which is essential for managing sessions.

- Metacognitive skills to reflect on the cognizing of others consequent to (in)actions.

To observe the producer taking decisions, our investigation targets actions associated with these skills since in real-world activities, decisions are not always neatly framed as decisions.

\section{An interdisciplinary approach}

A primary aim of this study is to find ways to observe a producer's meta-cognizing without having to ask him about how he (thinks he) thinks. But, artistic behaviour is extremely susceptible to observer effects and influences from the physical environment in which creation happens. Even in un-observed music production scenarios, musicians often feel inhibited just knowing that their performances will be recorded and repeatedly scrutinized by collaborators and audiences. To date, most research on music production comes from musicologists conducting ethnographic research while cognitive science and NDM often favour more constrained, empirical approaches. However, Suchman's (1987) "situated action" and Hutchin's (1995) "distributed cognition" frameworks illustrate how workplace studies have utilized ethnographic methods. Both these frameworks maintain a lasting influence in workplace analyses and decision-making research. (Sellberg and Lindblom 2014) This study utilizes data collected for ethnographic research in music production (i.e. session videos, audio recordings, field notes, etc.), but developed a method of analysis that contributes to both decision-making research as well as socio-cultural musicology.

Rogers suggests that the value of workplace study methods is determined by their "descriptive" and/or "application power" (Rogers 2004 via; Sellberg and Lindblom 2014). According to Sellberg and Lindblom (2014), the applicability of a method may be determined by considering the "theoretical depth"; "level of analysis"; "level of structure"; "type of process" and "duration" (to develop domain expertise and subsequently analyse) (Sellberg and Lindblom 2014 , p. 485). A primary goal of this study is to bridge the methodological gaps between decision-making research/ cognitive science and sociomusicology, and so the depth of historical analysis, "i.e., how and why a certain activity is performed in such way and how certain artefacts are developed over time used in that work practices" (Sellberg and Lindblom 2014, p. 485), is purposely limited. We are still able to affirm what is already known about the producer's role, common production practices and stylistic conventions, areas that musicology has already developed sufficient methods for investigating. In addition, for this research, we needed a descriptive method that revealed the specific information and considerations pertinent to the producer while he assessed situations and predicted events, and that factored into his decision-making. Our challenge is to identify this information and these considerations, whilst also retaining the naturalistic quality of the production process. In developing our approach, we extended the methodologies typically utilized in socio-cultural musicology, seeking not only to gain new insight into the producer's thinking, but also, new insight into production as a "type of process". Music production involves numerous iterative processes, but the sequence and duration of each constituent process is unpredictable and varies production-to-production. Sellberg and Lindblom's (2014) "duration" was not a primary a consideration. Both authors possess deep, applied domain expertise and this was employed in the identification of decision events.

\section{Recording studio data}

The data were originally compiled for a study on creative systems (Thompson 2016) in the recording studio. Thompson documented three recording sessions occurring over consecutive days, all part of the same recording project. Although conducting a sociomusicological study, Thompson's approach to data collection was not dissimilar to those used in cognitive work analysis, for example, FarringtonDarby and Wilson's (2009) video ethnography of rail network controllers. Furthermore, Thompson generally uses a "creative systems model" framework for analysis. Thompson 
was present at these sessions (as is common in sociomusicological research). He collected video and audio recordings, made field notes and conducted semi-structured interviews with the project's record producer. These same materials have been analysed again here, but with a completely different set of research questions and methods. The sessions took place at Elevator Studios, a well-known commercial studio in Liverpool. All participants volunteered to provide these data for research, and have agreed to the disclosure of their first names (Table 1). Through a research grant, Leeds Beckett University funded the studio time and the services of the professional producer and professional engineer involved. However, none of the participants were under any obligation whatsoever to deliver any specific quantity or kind of recorded content and all rights to the music and recordings belong solely to the musicians involved.

The activities of and interactions amongst the participants were captured using unobtrusive CCTV-style video cameras. They transmitted signals wirelessly to a computer where each perspective was recorded simultaneously and time stamped. Each camera had a microphone but to capture higher quality audio Dictaphones were placed near the recording console in the control room. The audio was transcribed. For this study, ten video clips from across all three sessions were selected, all were under $10 \mathrm{~min}$ in duration. Each clip features the producer interacting, reviewing recorded performances and mixes, and/or taking decisions at creative junctures.

The transcriptions of these clips provide the data for the analyses. No visual information was used, reducing the overall complexity of the experiment and minimizing inference and interpretation. Admittedly, visual cues provide evidence for emotion and the qualities of subjective experiences and, by removing them, the analysis perhaps biases decisions and metacognitive judgments that utilize technical information or semantic and formally acquired knowledge. The analysis did not investigate the role of emotion and "gut feelings" specifically, but did consider how the producer utilizes experience and the recognition of scenarios generally.

Table 1 Participants

\begin{tabular}{ll}
\hline Name & Role \\
\hline Marc & Record producer \\
Darren & Engineer \\
Paul & Lead vocalist/songwriter \\
Phil & Drummer \\
Mike & Lead guitarist \\
Rory & Trumpet player \\
Chris & Bass player \\
Nick & Saxophone player \\
Jess & Backing vocalist \\
\hline
\end{tabular}

The session participants are included in Table 1 .

\section{Coding method and first analysis}

Two analyses were performed on the dataset. Findings from the first analyses informed the second. The dataset includes producer comments from all 3 days of sessions. Metacognitive processes potentially influence the producer's decisionmaking in various ways throughout a production. So, the first methodological challenge was to determine what kinds of metacognitive reflection might be observable in the data. With categories for the skills, discriminations, judgements and tasks typically associated with the producer's role that are likely to be enabled by metacognition, we combed the producer's comments for examples, insights into how decisions were taken or actions performed and indicators of what information informed decisions. Then for the analysis of these comments, the following set of codes was devised. We took coded examples to be indicators that metacognitive reflection had happened.

- Valuations (of artefact attributes and actions that render attributes).

- Structuring of the perceptual problem space, physically and mentally controlling what is heard or considered to aid judgments.

- Applying strategies for problem-solving, that is, a justification of a specific approach to problem-solving, or an explanation of choice about potential actions.

- Management of how the session will progress towards its goals. These actions operate at levels that are not directly connected to perceptual attributes of the recording, for example, organizing problems to be solved on the way to session goals. It could involve inferring the cognizing of others to understand or manage their actions, thereby informing how a course of action is chosen.

- A direct reference to the producer's own thinking processes (i.e. I am thinking this because...).

It is vital to emphasise, again, that these are indicators that metacognitive reflection was highly likely to have happened around these comments (in many cases prior to the creative juncture moment of choice). The codes are not themselves indicative of specific kinds of metacognitive reflection.

The producer decides within a context and, for the purposes of this study, the definition of the context was limited to those attributes mentioned by the producer in the transcript or that are otherwise discernible from the transcripts. This included the participants collaborating in the session; the physical separation of the studio's live recording and control rooms; features of the console as 
mentioned by the producer; perceived physical attributes of the environment (if mentioned or discernible); the availability of microphones and signal processors mentioned by the producer, engineer or other collaborators; prior knowledge and experience that the producer divulges during interactions. Within each interaction, each of the producer's utterances was interpreted and categorized within the context of what has been said previously within that interaction. For the purposes of this study, each video clip was analysed as an instance of interaction or a creative juncture. That choice is not without its problems, but that debate lies outside the scope of this study. Only the producer's (Marc) comments were coded, but in the context of others' comments and activities in the environment.

The producer made 73 comments captured across the 10 video clips. Some comments contained more than one indicator of metacognitive reflection. In the 73 comments analysed, there were 30 indicators. Table 2 shows examples.

The frequencies of occurrences are broken down in Table 3.

There is not enough data in this first analysis to draw any conclusions about the significance of the distribution; furthermore, at this stage, there would be no way to ascertain if a significant pattern was idiosyncratic to this producer or generalizable across producers. However, given how the producer's expertise has been previously characterized in the musicology literature, the distributions are not surprising. Nevertheless, the confirmation helps to build conceptual bridges between cognitive and socio-musicological approaches. The socio-musicological literature's often functional structuring of the production problem space similarly facilitates evaluations. Since the method applied thus far identified numerous examples of actions enacted with specific ends in mind, the next step in analysis assumed that the producer's strategies are applied to realize certain values. Digging into those values offered deeper knowledge about the role of metacognition.
Table 3 Frequency of indicators

\begin{tabular}{lc}
\hline Code/indicator & $\begin{array}{l}\text { Frequencies } \\
\text { of occurrence }\end{array}$ \\
\hline Valuations & 8 \\
Structuring of the problem space & 10 \\
Applying strategies for problem-solving & 9 \\
Management & 2 \\
Direct reference (to cognition and action) & 1 \\
\hline
\end{tabular}

\section{Second analysis}

Continuing on from the first analysis, another code was created for the same set of comments and interactions. This code was devised to examine studio interactions that are particularly interesting for socio-cultural musicology, and where NDM can provide new understanding regarding what information a producer utilizes, how factors are balanced in production decisions, information is integrated, and how the producer's decisions relate to the work of other collaborators. The second code consisted of four categories of comment: drawing from experience, controlling the perceptual environment, getting into the band's head, and descriptions of a strategy. Again, these categories were selected because they are very likely to involve metacognitive reflection. In this analysis, rather than looking for frequencies of occurrences, examples from each category were deconstructed to reveal how the producer self-manages his decisions, interactions within and in response to the production (perceptual) environment; and how decisions and actions sustain collaboration (i.e. getting into the band's head). Drawing from experience, where his comments suggest some conscious reflection around experiences, implies that the producer has recognized a scenario and is considering how to adapt it. Stated strategies reveal what information has been considered and expectations about outcomes. The deconstructions discussed below tie together concerns of NDM and sociocultural musicological research. All quotes are attributed to the producer unless otherwise noted.

Table 2 Examples of indicators

\begin{tabular}{|c|c|}
\hline Code/indicator & Example \\
\hline Valuations & "I normally have it slightly to one side?" (Day 1) \\
\hline Structuring of the perceptual problem space & $\begin{array}{l}\text { "[J]ust bring that in, just those long notes. It might be interesting, it might just open the track up a } \\
\text { bit more." (Day 2) }\end{array}$ \\
\hline Applying strategies for problem-solving & $\begin{array}{l}\text { "Shall we see what the close are like first? Erm, otherwise, we could try a bit of distance after- } \\
\text { wards but yeh, that might lend more to the problem of spillage." (Day 1) }\end{array}$ \\
\hline Management & "But, that's not always best for the session is it?" (Day 2) \\
\hline Direct reference (to cognition and action) & "[H]ell what have I done? But you can’t say it out loud [laughs]." (Day 2) \\
\hline
\end{tabular}




\subsection{Drawing from experience}

On Day 1, there was a discussion amongst Marc, the producer, and various other band members about kick drum sounds. The central theme focuses on the benefits of replacing (through technical means) the kick drum sound captured on the recording with a pre-recorded sample. The rhythmic performance and some of the dynamics are retained, but the timbre is altered. It is not possible to have such a conversation without reflecting on previously acquired knowledge and pre-established biases.

Chris-_It's a bit too Metallica if it's on the beater..."

Marc_-"That's it yeh, I'm not a big fan of that."

Paul_-"or Lars Alrich."

Rory-"He uses triggers doesn't he?"

Marc- "Well, I have a confession. I use samples myself but I augment the samples with the recorded sound."

Rory-"Why?"

Marc- "Just to give it a little more punch and presence but it's because I like my mixes to be radio friendly and you can do great recordings with like, y'know, with that kind of stuff but to get it radio friendly and to get it within those band of frequencies. So there's a beat there if you want to dance to it in the kitchen."

Here the producer's values are revealed as preferences for radio friendliness. Those preferences were informed by prior experiences, when some attributes of kick drum sounds were chosen over others. Those same attributes were singled out for attention, appraisal and evaluation again here. Valuing attributes necessitates reflecting on popular music generally with the goal of furthering understanding the value of some attributes over others. He did not just learn by listening. He chose to use his listening as a means to solve the problem of radio-friendliness. He chose this problem over other problems such as the clarity of the beater.

- "That's it yeh, I'm not a big fan of that"

- "punch and presence"

- "radio friendly"

- "band of frequencies"

Certainly, it is plausible though unlikely that he favours samples because, for example, another producer favours them. Regardless, there is still a process of deciding how to decide based on values shaped by more than the immediate context. He explains his model. He explains what attributes he thinks should be emphasised in a production decision to create radio friendliness. Generalizations require comparisons of many cases and many approaches to attaining the qualities on which his model is based: a "band of frequencies" and "punch and presence".

At the moment this conversation is happening, the producer and engineer are negotiating the kick drum sound for the project in front of them. In popular music today, kick drums are often replaced with samples. Here, the decision is not merely procedural. The recorded sound is appraised through their individual preferences, biases, knowledge, listening skills and prior experiences. To lead decision-making, the producer considers his actions: what should the goal be, why, how can technical decisions in the present context realize meta-goals? In this negotiation with the engineer, this thinking is likely informed by the side conversation about radio-friendliness. These attributes are in the producers' mind. They have been declared as prior knowledge. He has justified their value, and admitted how he has realized them in practice.

All this implies the producer has metacognitive skills to consider the values of attributes in specific contexts and the value of different actions to achieve those attributes, and adapt decisions and actions accordingly. If music production were a context where the choices could be quantified and defined consistently, for example, in terms of specific frequencies at specific sound pressure levels and in particular combinations, it would be easier to apply classical, value choice, maximising gain decision-making models to what was observed here. The producer's choices could be explained as what gets him as close as possible to ideal values. However, a kick drum sound can never be quantified in these terms. Which band of frequencies, what sounds punchy and present are always determined relative to a musical context, a specific listening environment and its technical affordances, the social dynamics of a session, events pertinent to the field of music production, current social trends at large, etc. From a socio-musicological perspective, making a popular music recording "radio friendly" is not simply a technical matter. There are numerous indicators from the conversation excerpt above that suggest it involves balancing technical, musical and cultural concerns that are outside the immediate context of the recording studio, for example, the concerns of operatives within radio broadcast industry (Osborne 2012, p. 124).

The producer's actions could be interpreted as maximising what he prefers, working towards what he likes (a gut feeling), but the social context of the studio makes this unlikely. The producer works for clients, who have their own preferences, biases and goals. If these are not factored into the producer's decisions and he does not strike some balance of preferences, perspectives and knowledge in the room, he is unlikely to work as a producer for very long. Having a preference is not the same as awareness of the preference and reasoning about optimal ways to achieve preferred results in the current situation. For all these reasons, it is not possible to eliminate deciding how to decide from decisionmaking in music production. And, although it is not possible to directly observe the producer reflecting in the session, it can be inferred that reflecting on his mental models and 
processes would supply reasons and intention that can guide how he goes about making his appraisals.

\subsection{Controlling the perceptual environment}

The greatest number of indicators in the first analysis fell under the category of structuring of the problem space. Specifically, they related to physically framing what could be heard and, therefore, could inform decisions. Unsurprisingly, these comments were associated with requests to the engineer to mute or unmute audio channels, or otherwise adjust, something on the recording console. "Can you turn it down a little bit so the speakers aren't stressing?" (Day 1). "[J]ust bring that in, just those long notes. It might be interesting; it might just open the track up a bit more." (Day 2). This technique for evaluation is so common in music production that it is easy to overlook its significance in the decisionmaking process, and also the importance of metacognition in formulating these requests. Randomly or arbitrarily asking for tracks to be muted and unmuted serves no purpose. Thinking about problem-solving must precede the selection of appropriate and informative subgroups. It is worth noting that the ergonomic/technical configuration of the recording studio, the design of the recording console and monitoring system (uniquely) enables instantaneous physical manipulation of the listening environment-and thus direct manipulation of the decision-making space. From a socio-cultural musicological perspective, controlling the perceptual context helps the producer to make decisions with confidence; it is epistemic for him as well as any other participants within earshot.

\subsection{Getting into the band's head}

The next category was 'getting into the band's head'. In the first analysis, inferring the thinking of others fell under the category of management because others' thoughts, values, biases and skills are constraints in the choice of a strategy. They also limit how a strategy may be realized. "Well, yeh. I'm just thinking of Paul (the singer) all the time see because it's what he wants isn't it?" (Day 2). On Day 3, the producer said, "I think these ideas need to settle with you guys, when you've had a bit of distance from the last few days...". Thinking about what the singer wants or what the band's thinking needs involves reflecting on another's thinking, considering their goals, their perceptions and their problem-solving — with the aim of optimizing the producer's own decisions and actions. However hard it is for the producer to define those wants, it is almost impossible for an observer to (objectively) tease apart the singer's actual wants from the producer's understanding of them. Nevertheless, there is strong evidence that metacognitive processes were involved to encode the problem of understanding another's thinking. It also requires metacognition to strategically influence those thoughts, for example, by controlling the time allocated to make decisions.

Interestingly, in a post-session interview (conducted for Thompson 2016), the producer remarked, "My role was helping the band to realise what they heard in their heads". The producer elicited feedback through questions for the purposes of 'getting into the band's head'. Specific information was sought, with the intention of utilizing that information in his own decision-making, most likely integrating it with other forms of information. To formulate questions that would elicit the needed information, the producer had to draw from his previous musical, technical, social and cultural knowledge and experience. The band's preconceptions and expectations of the production process and the intended outcome of the recording provided information about goals, potentials and constraints, but again, producers have expertise other collaborators do not. So, the band's conceptions alone are insufficient representations of the decision-making task.

\subsection{Descriptions of strategy}

Finally, we found 'descriptions of a strategy'. The producer's comments revealed that he had specific strategies for problem-solving in mind, even if he did not always reveal the strategy itself, for example, "Well no I can fix that, possibly". The producer uses his prior knowledge and experience of previously encountered problems and recognizes scenarios (Day 2). There were also patterns to his inquiries that suggest there is an end goal in mind and that he has considered ways to achieve those ends. "Shall we see what the close are like first? Erm, otherwise, we could try a bit of distance afterwards but yeh, that might lend more to the problem of spillage." (Day 1). In this case, if the first action does not provide the desired value, it nevertheless results in information that will inform subsequent choices. To further his problem-solving, the producer takes steps to determine the extent of problems.

Each of the four deconstructions above provide some clues into what information a producer utilizes, balancing factors in production decisions and integrating information, and how the producer's decisions relate to the work of other collaborators. However, the complexity of each of these interactions, the number and diversity of factors that contribute to each decision, is still, from a methodological perspective, unwieldy. Nevertheless, using this kind of ethnographic data made it possible to find numerous examples of skills, discriminations or judgements typically ascribed to the producer's role, and there was evidence that exercising these skills or performing these tasks necessitated metacognitive reflection. For a more a more in-depth analysis of how information was utilized it would be necessary to 
deconstruct specific decisions. This may mean sacrificing some ecological validity or complementing empirical observations with other ethnographic approaches. With enough data from this producer, it may be possible to find patterns in this producer's decision-making (if they exist). And, with enough data from many producers across assorted sessions, it may be possible to find generalizable patterns in producers' decision-making more broadly.

\section{Discussion and a model of decision-making in the recording studio}

Because studio production is so ill structured and risky, even for those scenarios where a producer has time to analyse and weigh decisions and outcomes, it is difficult to apply classical decision-making models to the context of the recording studio, whereas the NDM framework accommodates the inherent pressures and uncertainty, and indeed, the fact that many outcomes can scarcely be predicted at all. This study shows the producer recognizing situations and problems, as in RPD. But perhaps more interesting is how production scenarios, once recognized, are adapted. Adapting creative solutions entails comparisons among artistic artefacts, and there are innumerable, useful ways a producer may choose to make those comparisons. Additionally, producers sometimes seek solutions that keep an artefact consistent with genre norms and at other times, novelty is valued. The results presented here suggest that metacognitive judgements are an integral part of deciding how, when and why solutions and decisions conform or innovate.

This study captured the varied information and knowledge needed to take production decisions as well as the diversity of those decisions. It showed that production decisions are contingent on pragmatic matters such as time and available technology, values for creativity and contributors' biases and preferences, as well as, factors external to the production environment. Figure 1, below, illustrates this complexity. A produced artefact can be characterized by the sum of the outcomes $\left(\mathrm{O}_{1}+\mathrm{O}_{2}+\cdots \mathrm{O}_{x}\right)$ of decisions and actions taken at various junctures $\left(\mathrm{J}_{1}+\mathrm{J}_{2}+\cdots \mathrm{J}_{x}\right)$. Each time the producer decides, at every creative juncture, the end-product becomes a little more definable, if not always more satisfactory. Thus, each decision either provides more information or makes it possible to use available information with greater intent and precision. Every decision is framed by various potentials for making the product acceptable, which are influenced by technical affordances and expressive and commercial opportunities and by constraints such as time and finances and artistic and technical limitations.

The model further shows how different types of decisionmaking processes are applied at different decision junctures.
The availability of time is clearly only one constraining factor involved in how decisions are taken. The kind of action and outcome deemed valuable at that juncture will also influence how to decide, as will the assessment of an opportunity for novelty or norm. Part of what makes creative decisionmaking so complex is that it requires deciding when to be creative. The dotted lines (in Fig. 1) illustrate where factors are weighted dynamically and illustrate where metacognition can enable the producer to choose and weigh factors, utilizing and integrating varied types of information, including information available in the production environment and previous acquired experience and knowledge. Metacognitive reflection provides a useful explanation for how this integration happens. Metacognition enables the producer to judge the importance of information and to identify actions that conform to the producer's and other collaborators' biases and the producer's beliefs about the client's and audience's expectations. Although classical decision-making models can be used to capture components of common practice or innovative procedures in music production, they cannot fully explain how practices or procedures are adapted for a specific use context. Metacognition helps in this regard to begin to provide a vital part of this explanation.

\section{Conclusions}

Artistic working environments are underrepresented in the NDM literature. This study investigated NDM in a professional, studio-based, music production environment, and specifically the decisions of the record producer. The study aimed to investigate the macrocognitive work environment of the recording studio by employing both cognitive sociocultural musicological approaches to observe the producer's metacognitive reflections. This was a means to deepen understanding about the producer's decision-making and problem-solving. Utilizing data (i.e. session videos, audio recordings, field notes, etc.) originally collected for an ethnographic study of a music production, this study involved two analyses. The first involved coding recording session transcripts. The producer's comments were categorized as examples of decisions. The method tested did identify numerous examples of actions enabled by metacognitive processes. Based on the first analysis, another code was created for the same set of comments and interactions. This code was devised to examine studio interactions that are of interest to socio-cultural musicology, where NDM can help elucidate what information producers utilize, how they balance factors in production decisions, integrate information, and how the producer's decisions relate to the work of other collaborators.

Preliminary findings from both analyses shed light on how the producer in this study self-manages his decisions 
Fig. 1 Flow of information and components of decisions for creative junctures in music production
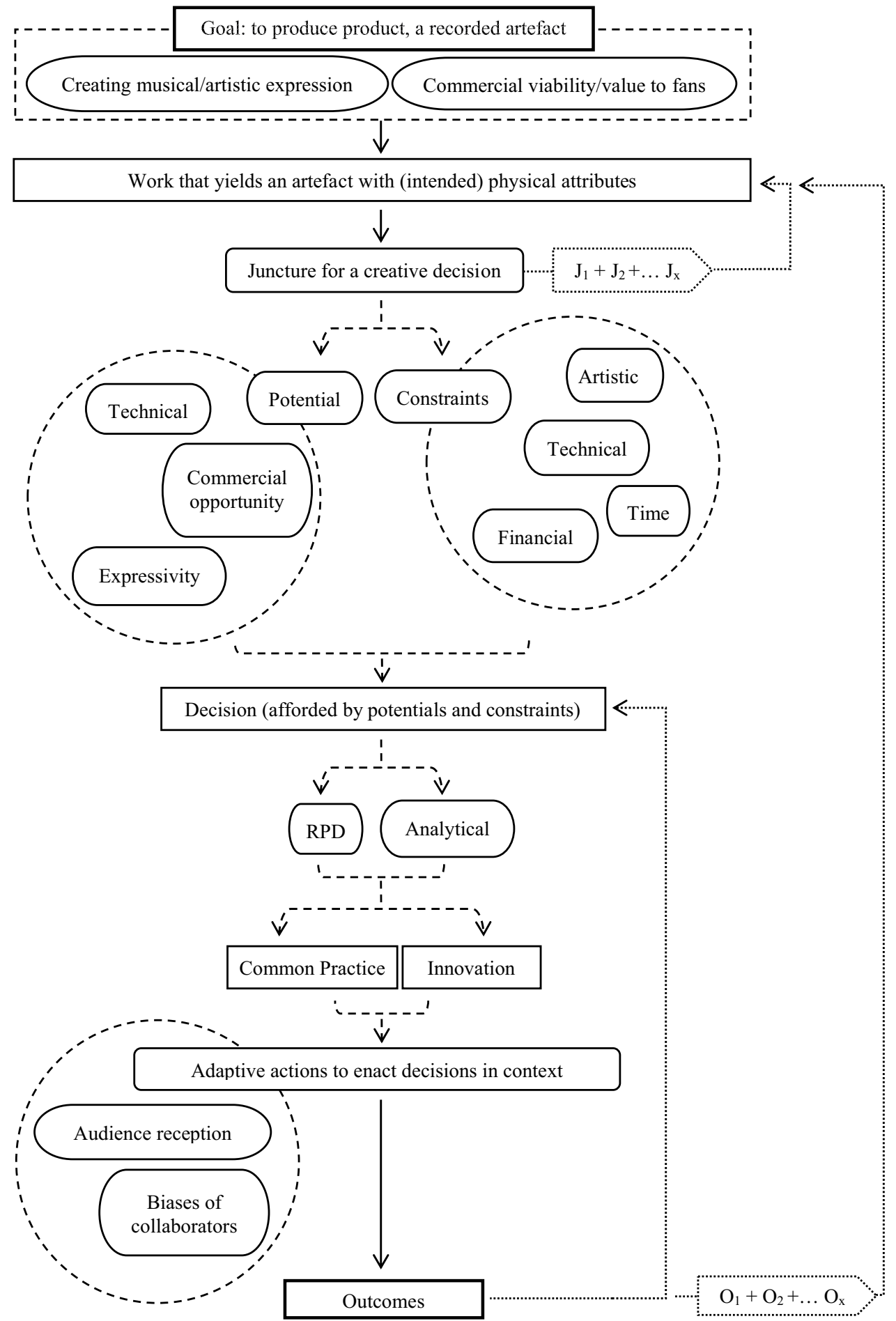

and his interactions with and in response to the production environment, how decisions and actions sustain collaboration, how experience is utilized to identify scenarios and choose actions, and the kinds of strategies employed and their expected outcomes. Although methodologically cumbersome, using ethnographic data made it was possible to find numerous examples of skills, discriminations and judgements that are typically ascribed to the producer's role. Furthermore, there was evidence that exercising these skills or performing these tasks suggest certain kinds of metacognitive reflection.

Further work is needed in this area, specifically, to determine how exactly information is utilized by the producer in decision-making. This may mean sacrificing some 
environmental naturalness using more empirical methods and/or by utilizing additional, complementary ethnographic approaches such as document analysis. In any case, with enough data, it may be possible to find patterns within one producer's decision-making (if they exist), or with enough data from many producers across assorted sessions, it may be possible to find generalizable patterns in producers' decision-making more broadly.

Open Access This article is distributed under the terms of the Creative Commons Attribution 4.0 International License (http://creativeco mmons.org/licenses/by/4.0/), which permits unrestricted use, distribution, and reproduction in any medium, provided you give appropriate credit to the original author(s) and the source, provide a link to the Creative Commons license, and indicate if changes were made.

\section{References}

Cacciabue PC, Hollnagel E (1995) Simulation of cognition: Applications. In: Hoc J, Cacciabue PC, Hollnagel E (eds) Expertise and technology: cognition and human-computer cooperation. Erlbaum, Hillsdale, pp 55-73

Davidson J, Deuser R, Sternberg RJ (1994) The role of metacognition in problem solving. In: Metcalfe J, Shimamura AP (eds) Metacognition. MIT press, Cambridge, MA, pp 207-226

Farrington-Darby T, Wilson JR (2009) Understanding social interactions in complex work: a video ethnography. Cogn Technol Work 11(1):1-15

Feld S (2004) Communication, music and speech about music. In: Charles Keil \& Steven Feld (authors) music grooves. University of Chicago Press, Chicago, pp 109-150

Flavell JH (1987) Speculations about the nature and development of metacognition. In: Weinert FE, Kluwe RH (eds) Metacognition, motivation and understanding. Lawrence Erlbaum Associates, Hillside, pp 21-29

Hutchins E (1995) Cognition in the wild. MIT press, Cambridge, MA

Kirsh D (2009) Problem solving and situated cognition. In: Robbins P, Aydede M (eds) The Cambridge handbook of situated cognition. Cambridge University Press, Cambridge, pp 264-306
Klein GA (1993) A recognition-primed decision (RPD) model of rapid decision making. In: Klein GA, Orasanu J, Calderwood R, Zsambok CE (eds) Decision making in action: models and methods. Ablex Publishing, Westport, pp 138-147

Klein GA, Ross KG, Moon BM, Klein DE, Hoffman RR, Hollnagel E (2003) Macrocognition. IEEE Intell Syst 18(3):81-85

Klein G, Pliske R, Crandall B, Woods DD (2005) Problem detection. Cogn Technol Work 7(1):14-28

Koriat A, Levy-Sadot R (2000) Conscious and unconscious metacognition: a rejoinder. Conscious Cogn 9(2):193-202

Neuenfeldt K (2005) Nigel Pegrum, "Didjeridu-Friendly Sections," and What Constitutes an "Indigenous" CD: an australian case study of producing "World Music" Recordings". In: Green PD, Porcello $\mathrm{T}$ (eds) Wired for sound: engineering and technologies in sonic cultures. Wesleyan University Press,, Middletown, pp 84-102

Osborne J (2012) Radio head: up and down the dial of British radio, 2nd edn. Simon and Schuster UK Ltd, London

Patterson ES, Hoffman RR (2012) Visualization framework of macrocognition functions. Cogn Technol Work 14(3):221-227

Rogalski J (1999) Decision making and management of dynamic risk. Cogn Technol Work 1(4):247-256

Rogers Y (2004) New theoretical approaches for human-computer interaction. Annul Rev Inf Sci Technol 38:87-143

Sellberg C, Lindblom J (2014) Comparing methods for workplace studies: a theoretical and empirical analysis. Cognit Technol Work 16(4):467-486

Simon HA (1973) The structure of ill structured problems. Artif Intell 4(3-4):181-201

Suchman L (1987) Plans and situated actions. Cambridge University Press, Cambridge

The Beatles (1967) Sgt. Pepper's lonely hearts club band. Parlophone, UK

Thompson P (2016) 'Scalability of the Creative System in the Recording Studio'. In: McIntyre P, Fulton J, Paton E (eds) The creative system in action: understanding cultural production and practice. Palgrave Macmillan, Basingstoke, pp 74-86

Vyas D, van der Veer G, Nijholt A (2013) Creative practices in the design studio culture: collaboration and communication. Cogn Technol Work 15(4):415-443

Zak AJ (2001) The poetics of rock: cutting tracks, making records. University of California Press, Los Angeles 